American Journal of Applied Sciences 5 (1): 55-60, 2008

ISSN 1546-9239

(C) 2008 Science Publications

\title{
Transversely Cracked Beam's Finite Elements with a Hinged End
}

\author{
M. Skrinar and T. Pliberšek \\ University of Maribor, Faculty of Civil Engineering Maribor, \\ Smetanova 17, SI 2000 Maribor, Slovenia
}

\begin{abstract}
Two new beam finite-elements to be used for the transverse displacement analysis of slender beams with transverse cracks are presented where the derivations are based on a simplified computational model. The beam stiffness matrixes are presented in symbolic forms for beams with a single transverse crack and a hinge at one of the nodes. Since in the derivations the corresponding interpolation functions were implemented, the transverse displacements within the finite element can be afterwards obtained by introducing the discrete values of nodal displacements and rotations into presented analytical solutions. A numerical example concludes the material and shows that, although with considerably less computational effort than with 2D finite element meshes, the presented beam finite elements yield results that exhibit excellent agreement with the results from the huge $2 \mathrm{D}$ FE meshes. Due to the fact that the number of parameters describing the cracked beam structure is thus reduced to its minimum it can be expected that these elements could be efficiently implemented in inverse identification of cracks.
\end{abstract}

Key Words: Simplified computational model, beam stiffness matrix, transverse displacements

\section{INTRODUCTION}

Cracks are doubtlessly one of the most unfavourable and negative effects that might appear on a structure during its utilization. They should be detected as soon as possible as they may significantly decrease the global stiffness over the local reduction of flexural rigidity. Since in inverse identification of cracks only a limited amount of measured data is available, it is thus usually not possible to obtain the information of crack directly but as a result of some gradual and systematic computational model modifications. In order to make the computational model more adaptable and thus allow a faster analysis process, it is reasonable to neglect all the irrelevant information from the computational model. Therefore, in simplified model of a beam with a non-propagating crack the information about stresses could be ignored.

A simplified computational model for transverse displacement computations of a cracked beam, originally presented by Okamura et al. ${ }^{[1]}$ meets all the requirements stated above. The transverse crack was introduced as a rotational linear spring with stiffness $K_{r}$ connecting the uncracked parts of the element modeled as elastic sections. Okamura's computational model has also already been experimentally implemented regarding inverse identifications based on the structure's dynamic response. By using controlled forced vibrations and measured displacements at two selected points the location of the crack has been identified on a cantilever ${ }^{[2]}$. This approach was also verified experimentally. Implementing measured eigenfrequencies only, the location of the crack has been experimentally identified on a single beam with free ends ${ }^{[3]}$. In both approaches the differential equation of motion was solved, and the location of the artificially introduced crack was identified over the rotational spring stiffness value $K_{r}$ instead of the crack depth $d$ itself.

The research interest was further oriented towards a numerical solution for transverse displacements' computation implementing beam finite elements. In the first step, the stiffness matrix of a beam finite element with a single transverse crack and clamped ends, SCBFE, was given in symbolic form ${ }^{[4]}$. The derivation of this element implemented static interpolation functions. Further progress was achieved when for a static finite element with an arbitrary number of transverse cracks, a numerical procedure was presented for the numerical computation of stiffness matrix ${ }^{[5]}$. Furthermore, the effect of transverse displacements due to axial tensile loads on single-sided transversely cracked elements was also studied on single element structures $^{[6-9]}$.

The unfavourable (especially from the inverse identification point of view) problem of the increasing number of static beam finite elements along each structural element in dynamic analysis, has been solved by dynamic finite element of a slender beam with a single transverse crack, DSCBFE, derived by
Corresponding Author:
Matjaž Skrinar, University of Maribor, Faculty of Civil Engineering Maribor, Smetanova 17, SI 2000 Maribor, Slovenia, Tel: + 38622294 358, Fax: + 38622524179 
implementing dynamic interpolation functions, yielding frequency dependent stiffness and mass matrixes ${ }^{[10]}$.

The already presented solutions allowed modeling of the hinges at supports only where the effect of a hinge was achieved by preventing transverse displacements. For hinges at structural elements' joints or within the structural elements, additional standard uncracked beam finite elements had to be implemented, unfavourably increasing the computational model.

\section{MATERIALS AND METHODS}

Mathematical formulation and derivation steps: The derivation of both new beam finite elements is based on the mathematical model of transversely cracked beam as given by Okamura et al. ${ }^{[1]}$. The uncracked parts of the element are modeled as elastic parts to the left and to the right of the crack, and the crack itself is modeled by a rotational spring with stiffness $K_{r}$. The distance $L_{I}$ from the left end of the element defines the position of the crack and the uniform depth of the crack is denoted with $d$. The rotational spring stiffness $K_{r}$ is a function of the height of the uncracked cross-section $h$, the relative depth of the crack $\delta=d / h$, and the product of Young's modulus $E$ by the moment of inertia of the uncracked cross-section $I_{z}$, i.e. flexural rigidity EI. Okamura's genuine definition for rotational stiffness also takes Poisson's ratio $v$ into account. The remaining definitions can be found in the studies about the implementation of Okamura's computational model ${ }^{[11-}$ 16].

Two nodes are implemented for each considered finite element in order to describe the transverse displacements, each of them at one end of the element. The finite element has four degrees of freedom altogether: transverse displacement $Y_{1}$ and rotation $\Phi_{1}$ at the left end (starting node), as well transverse displacement $Y_{2}$ and rotation $\Phi_{2}$ at the right end (ending node). Upward translations are taken as positive and counterclockwise rotations are also taken as positive.

For slender beams and under assumption of Bernoulli-Euler's hypothesis, axial displacements $u$ can be expressed from transverse displacements $v$ as $(y$ represents the distance from neutral axis):

$u(x, y)=-y \cdot \frac{d v(x)}{d x}$

The element's strain energy consists of the contributions from both elastic parts as well as spring:

$$
\begin{aligned}
& U=\frac{1}{2} \cdot \int_{V} \sigma_{x} \cdot \varepsilon_{x} \cdot d V+\frac{1}{2} \cdot K_{r} \cdot \Delta \varphi^{2} \\
& =\frac{1}{2} \cdot \iint_{L} \sigma_{A} \sigma_{x} \cdot \varepsilon_{x} \cdot d A \cdot d x+\frac{1}{2} \cdot K_{r} \cdot \Delta \varphi^{2}
\end{aligned}
$$

where $\Delta \varphi=\left.\left(\varphi_{1}-\varphi_{2}\right)\right|_{x=L_{1}}$ is the difference of rotations of the cross sections to the left and to the right of the crack. Introducing linear relation between normal strains $\varepsilon_{x}$ and normal stresses $\sigma_{x}$, Eq. (2) thus turns into

$U=\frac{1}{2} \cdot \int_{x=0}^{L} E \cdot I_{z} \cdot\left(\frac{d^{2} v}{d x^{2}}\right)^{2} \cdot d x+\frac{1}{2} \cdot K_{r} \cdot \Delta \varphi^{2}$

However, since the crack separates the beam into two elastic parts, the transverse displacements must be separately given for the parts to the left $\left(v_{1}\right)$ and to the right $\left(v_{2}\right)$ of the crack. They are assumed in the form of the complete polynomials of the third degree with altogether eight unknown constants:

$$
\begin{array}{ll}
v_{1}(x)=\alpha_{1}+\alpha_{2} \cdot x+\alpha_{3} \cdot x^{2}+\alpha_{4} \cdot x^{3} & \left(0 \leq x \leq L_{1}\right) \\
v_{2}(x)=\beta_{1}+\beta_{2} \cdot x+\beta_{3} \cdot x^{2}+\beta_{4} \cdot x^{3} & \left(L_{1} \leq x \leq L\right)
\end{array}
$$

The unknown coefficients $\alpha_{1}, \alpha_{2}, \alpha_{3}, \alpha_{4}$ and $\beta_{1}, \beta_{2}, \beta_{3}, \beta_{4}$ are obtained from boundary and continuity conditions. Boundary conditions depend on actual situations at both ends of the analyzed element and, therefore, are given separately with each derivation. However, the continuity conditions at the crack location $\left(x=L_{l}\right)$ are identical for both finite elements under consideration. For the beam with constant flexural rigidity the continuity conditions are given as the equality of displacement, the condition for the discrete increase of rotations, the equality of bending moments, and the equality of shear forces:

$v_{l}\left(L_{l}\right)=v_{2}\left(L_{l}\right), v_{l}{ }^{\prime}\left(L_{l}\right)+\frac{E I}{K_{r}} \cdot v_{l}{ }^{\prime \prime}\left(L_{l}\right)=v_{2}{ }^{\prime}\left(L_{l}\right)$,
$v_{l}{ }^{\prime \prime}\left(L_{l}\right)=v_{2}{ }^{\prime \prime}\left(L_{l}\right), v_{l}{ }^{\prime \prime \prime}\left(L_{l}\right)=v_{2}{ }^{\prime \prime \prime}\left(L_{l}\right)$

With all unknown coefficients determined, the transverse displacements of considered part of the element are further expressed with the vector of unknown nodal displacements and rotations $\{q\}=\left\{Y_{1}, \Phi_{1}, Y_{2}, \Phi_{2}\right\}^{T}$ as:

$v_{l}(x)=\left\{N_{11}, N_{12}, N_{13}, N_{14}\right\} \cdot\{q\}=\left\{N_{l}(x)\right\} \cdot\{q\}$

$v_{2}(x)=\left\{N_{21}, N_{22}, N_{23}, N_{24}\right\} \cdot\{q\}=\left\{N_{2}(x)\right\} \cdot\{q\}$

Finally, the stiffness matrix of the finite element can be obtained by: 


$$
\begin{aligned}
& {[K]=\int_{x=0}^{L_{l}} E \cdot I_{z} \cdot\left\{N_{l}{ }^{\prime \prime}(x)\right\}^{T} \cdot\left\{N_{l}{ }^{\prime \prime}(x)\right\} \cdot d x} \\
& +\int_{x=L_{1}}^{L} E \cdot I_{z} \cdot\left\{N_{2}{ }^{\prime \prime}(x)\right\}^{T} \cdot\left\{N_{2}{ }^{\prime \prime}(x)\right\} \cdot d x+ \\
& \left.K_{r} \cdot\left(\left\{N_{I^{\prime}}(x)\right\}\right\}_{x=L_{I}}-\left.\left\{N_{2}{ }^{\prime}(x)\right\}\right|_{x=L_{I}}\right)^{T} \\
& \cdot\left(\left\{N_{I^{\prime}}(x)\right\}_{x=L_{I}}-\left\{N_{2}{ }^{\prime}(x)\right\}_{x=L_{I}}\right)
\end{aligned}
$$

while the corresponding load vector due to a distributed load $\mathrm{q}(\mathrm{x})$ is obtained as:

$$
\{F\}=\int_{x=0}^{L_{l}} q(x) \cdot\left\{N_{l}(x)\right\} \cdot d x+\int_{x=L_{l}}^{L} q(x) \cdot\left\{N_{2}(x)\right\} \cdot d x
$$

Beam finite element with a hinge at the left SCBFEHC: For the finite element SCBFEHC (Fig. 1), the boundary conditions required to obtain the interpolation functions embody three kinematical conditions: unknown discrete transverse displacement in the left node $Y_{1}$, displacement $Y_{2}$ and rotation $\Phi_{2}$ at the right node of the element, as well as mechanical condition mathematically describing the presence of the hinge in the beginning node:

$$
v_{l}(0)=Y_{1}, v_{l}^{\prime \prime}(0)=0, v_{2}(L)=Y_{2}, v_{2}{ }^{\prime}(L)=\Phi_{2}
$$

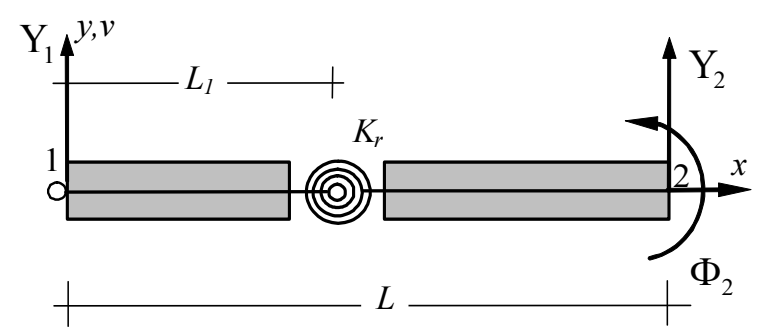

Fig. 1: Element SCBFEHC with corresponding degrees of freedom

Interpolation functions that satisfy boundary and continuity conditions are:

$$
\begin{aligned}
& N_{11}=\frac{2 \cdot L^{3}+6 \cdot L_{I}^{2} \cdot \psi-3 \cdot L^{2} \cdot x-6 \cdot L_{I} \cdot \psi \cdot x+x^{3}}{2 \cdot L^{3}+6 \cdot L_{I}{ }^{2} \cdot \psi}, \\
& N_{12}=0, N_{13}=\frac{3 \cdot L^{2} \cdot x+6 \cdot L_{I} \cdot \psi \cdot x-x^{3}}{2 \cdot L^{3}+6 \cdot L_{l}{ }^{2} \cdot \psi}, \\
& N_{14}=-\frac{x \cdot\left(L^{3}+6 \cdot L \cdot L_{I} \cdot \psi-6 \cdot L_{l}^{2} \cdot \psi-L \cdot x^{2}\right)}{2 \cdot L^{3}+6 \cdot L_{I}^{2} \cdot \psi}, \\
& N_{21}=\frac{(L-x)^{2} \cdot(2 \cdot L+x)}{2 \cdot L^{3}+6 \cdot L_{l}^{2} \cdot \psi}, \quad N_{22}=0,
\end{aligned}
$$

with $\psi$ representing the ratio between flexural rigidity $E I$ and rotational spring stiffness $K_{r}$. It can be easily verified that for the uncracked case (i.e. $K_{r}=\infty$ or $\psi=0$ ) presented interpolation functions become standard interpolation functions for the noncracked beam finite element for hinged - clamped boundary conditions. Finally, the stiffness matrix is

$$
\left[K_{H C}\right]=\frac{3 \cdot E I}{L^{3}+3 \cdot \psi \cdot L_{l}^{2}} \cdot\left[\begin{array}{cccc}
1 & 0 & -1 & L \\
0 & 0 & 0 & 0 \\
-1 & 0 & 1 & -L \\
L & 0 & -L & L^{2}
\end{array}\right]
$$

The corresponding load vector for a uniform transverse load $q$ over the entire element is further given as:

$$
\begin{aligned}
& \left\{F_{H C}\right\}=\frac{q}{8 \cdot\left(L^{3}+3 \cdot \psi \cdot L_{I}{ }^{2}\right)} \text {. } \\
& \left\{\begin{array}{c}
3 \cdot\left(L^{4}+4 \cdot L_{l}{ }^{3} \cdot \psi\right) \\
0 \\
5 \cdot L^{4}+24 \cdot L^{2} \cdot L_{l}{ }^{2} \cdot \psi-12 \cdot L_{l}{ }^{3} \cdot \psi \\
-L \cdot\left(L^{4}+12 \cdot L \cdot L_{l}{ }^{2} \cdot \psi-12 \cdot L_{l}{ }^{3} \cdot \psi\right.
\end{array}\right\}
\end{aligned}
$$

Beam finite element with a hinge at the right end SCBFECH: The finite element SCBFECH (Fig. 2) has a hinge at the right, ending node and, therefore, the kinematical boundary conditions embody unknown discrete displacement $Y_{l}$ and rotation $\Phi_{l}$ at the left node, as well as displacement $Y_{2}$ at the right node of the element. The fourth boundary condition mathematically describes the mechanical condition of the hinge's presence in the ending node:

$v_{l}(0)=Y_{1}, v_{l}{ }^{\prime}(0)=\Phi_{1}, v_{2}(L)=Y_{2}, v_{2}{ }^{\prime \prime}(L)=0$.

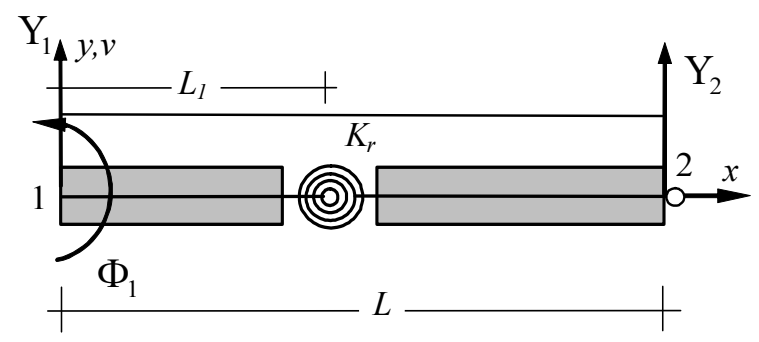

Fig. 2: Element SCBFECH with corresponding degrees of freedom 
Introducing the abbreviation

$$
\bar{L}=L^{3}+3 \cdot L^{2} \cdot \psi-6 \cdot L \cdot L_{l} \cdot \psi+3 \cdot L_{l}{ }^{2} \cdot \psi
$$

the interpolation functions that satisfy boundary and continuity conditions are:

$$
\begin{aligned}
& N_{11}=1-\frac{3 \cdot L}{2 \cdot \bar{L}} \cdot x^{2}+\frac{x^{3}}{2 \cdot \bar{L}}, N_{13}=\frac{3}{2 \cdot \bar{L}} \cdot x^{2}-\frac{x^{3}}{2 \cdot \bar{L}} \\
& N_{12}=x-\frac{3 \cdot L^{2}}{2 \cdot \bar{L}} \cdot x^{2}+\frac{L \cdot x^{3}}{2 \cdot \bar{L}}, N_{14}=0 \\
& N_{21}=\frac{L^{3}+3 \cdot\left(L-L_{1}\right) \cdot(L-x) \cdot \psi}{\bar{L}}+\frac{(x-3 \cdot L) \cdot x^{2}}{2 \cdot \bar{L}} \\
& N_{23}=\frac{3 \cdot\left(L-L_{1}\right) \cdot\left(x-L_{1}\right) \cdot \psi}{\bar{L}}+\frac{(3 \cdot L-x) \cdot x^{2}}{2 \cdot \bar{L}} \\
& N_{22}=\frac{3 \cdot\left(L^{3} \cdot x+\psi \cdot\left(L-L_{1}\right) \cdot(L-x) \cdot L_{1}\right)}{\bar{L}} \\
& +\frac{L \cdot(x-3 \cdot L) \cdot x^{2}}{2 \cdot \bar{L}}, N_{24}=0
\end{aligned}
$$

Finally, the stiffness matrix is

$$
\left[K_{C H}\right]=\frac{3 \cdot E I}{L^{3}+3 \cdot \psi \cdot L_{2}{ }^{2}} \cdot\left[\begin{array}{cccc}
1 & L & -1 & 0 \\
L & L^{2} & -L & 0 \\
-1 & -L & 1 & 0 \\
0 & 0 & 0 & 0
\end{array}\right]
$$

and the corresponding load vector for a uniform transverse load $q$ over the complete element is given as:

$$
\begin{aligned}
& \left\{F_{C H}\right\}=\frac{q}{8 \cdot \bar{L}} \cdot \\
& \left\{\begin{array}{c}
5 \cdot L^{4}+12 \cdot\left(L^{3}-L^{2} \cdot L_{I}-\cdot L_{I}{ }^{2}+L_{I}{ }^{3}\right) \cdot \psi \\
L \cdot\left(L^{4}+12 \cdot\left(L^{2} \cdot L_{I}-L \cdot L_{l}{ }^{2}+L_{l}{ }^{3}\right) \cdot \psi\right. \\
3 \cdot\left(L^{4}+4 \cdot\left(L^{3}-3 \cdot L^{2} \cdot L_{I}+3 \cdot L \cdot L_{I}{ }^{2}-L_{I}{ }^{3}\right) \cdot \psi\right. \\
0
\end{array}\right\}
\end{aligned}
$$

\section{RESULTS AND DISCUSSION}

In the presented example, a simply supported beam was considered. The length $L$ of the span was $10 \mathrm{~m}$, and the cross-section of the beam was a rectangle with dimensions $0.10 \mathrm{~m} / 0.20 \mathrm{~m}$. The Young modulus $E$ was $30 \mathrm{GPa}$, while the Poisson's ratio $v$ was taken as 0.3 . Two transverse cracks were induced, located at the distances of $3 \mathrm{~m}$ and $2 \mathrm{~m}$, from the left and right support, respectively. The selected depth of the cracks was $0.1 \mathrm{~m}$. This structure was selected because despite the presence of two cracks on the structure the reactions and the inner forces can be easily determined with the elementary static. Two load cases are considered here.
First load case: A concentrated vertical upward load $P=10 \mathrm{kN}$ was applied at a distance of $5.5 \mathrm{~m}$ from the left support, Fig. 3. The structure was modeled with three nodes (located at left end, under load and at right end), and both newly derived beam finite elements, connected at the position of the load. For the evaluation of rotational linear spring stiffness the definition given by Okamura was selected among all existing definitions due to the fact that it is the only one that takes the Poisson's ratio into account. The value of the rotational spring stiffness for the simplified model was therefore $K_{r}=3.14197572 \cdot 10^{6} \mathrm{Nm}$.

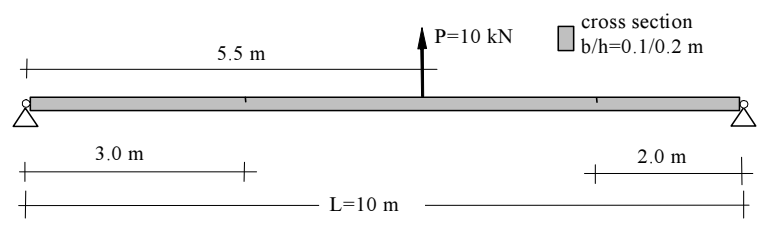

Fig. 3: Geometry of first load case

The model had 4 degrees of freedom but taking into the account the prescribed zero displacements (vertical displacement at both supports) the problem reduced to a system of just two linear equations with two unknowns (vertical displacement $Y_{2}$ and rotation $\Phi_{2}$ beneath the applied force). The unknowns were obtained from the following system (where the structure's global stiffness matrix $[K]$ was obtained from the element's stiffness matrixes):

$\left\{\begin{array}{l}Y_{2} \\ \Phi_{2}\end{array}\right\}=[K]^{-1} \cdot\{F\}=$

$\left[\begin{array}{cc}93437.753 & 93604.207 \\ 93604.207 & 2218980.180\end{array}\right]^{-1} \cdot\left\{\begin{array}{l}P \\ 0\end{array}\right\}$

$=\left\{\begin{array}{l}0.1117453 m \\ -0.0047138\end{array}\right\}$

Introducing the two obtained discrete values into the derived interpolation functions, the distribution of transverse displacements was obtained also along the longitudinal axis of the structure, Fig. 4. Figure 4 further shows the displacements obtained in discrete points by solving approximately 120,000 linear equations from the computational model where the complete structure was analyzed implementing a commercial finite element program COSMOS/M. The discrete crack approach was utilized to model the crack and the computational model consisted of 20,000 2D 8 noded quadrilateral plane finite elements.

The values for displacements for some representative points are summarized in Table 1. 
Table 1: Comparison of vertical displacements for the first load case

\begin{tabular}{lcc}
\hline Location $(\mathrm{m})$ of the point & \multicolumn{2}{c}{ vertical displacements $(\mathrm{m})$} \\
\hline & 2 beam FE & $20,0002 \mathrm{D} \mathrm{FE}$ \\
$\mathrm{x}=3.0$ (left crack) & 0.090717 & 0.090456 \\
$\mathrm{x}=5.0$ (midspan) & 0.112602 & 0.112390 \\
$\mathrm{x}=5.5$ (under the force F ) & 0.111745 & 0.111540 \\
$\mathrm{X}=8.0$ (right crack) & 0.068450 & 0.068182 \\
\hline
\end{tabular}

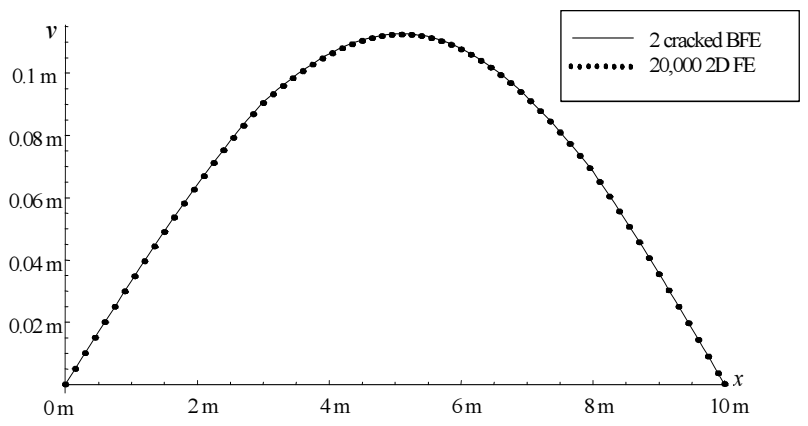

Fig. 4: Transverse displacements $v$ for first load case

Second load case: On the same structure a vertical upward uniformly distributed load $q=10 \mathrm{kN}$ over the whole span was applied. The structure was first modeled with both newly derived beam finite elements, however, now connected at the midspan. Similarly to the previous load case the analysis again reduced to a system of just two linear equations with two unknowns (vertical displacement and rotation at the midspan). In addition, also this load situation was analyzed implementing the computational model with 20,000 2D quadrilateral plane finite elements. Although in the first load case the agreement of the results was excellent for all point along the structure, in this situation the discrepancies of the displacements in the regions between the nodes become noticeable. The reason for this lies in the fact that the implemented interpolation functions for the derived finite elements are complete polynomials of the third degree, while the actual elastic curve of transverse displacements due to a uniform load is a complete polynomial of the fourth degree. Therefore, the structure was re-analyzed implementing three beam finite elements (a standard beam FE was inserted between the two cracked beam finite elements), with the lengths of $3.5 \mathrm{~m}, 5.0$ and $2.5 \mathrm{~m}$. This consequently increased the rank of system of linear equations to four, but also clearly reflected in the increased matching of the results, Fig. 5.

The displacements values for some representative points are summarized in Table 2 .

\section{DISCUSSION}

From Figures 4 and 5 it is clearly evident that only moderate discrepancies appeared when comparing the results from the appropriate simplified model with the results from the detailed $2 \mathrm{D}$ finite element mesh. The values presented in Tables 1 and 2 confirm that as the absolute maximum difference for displacement is smaller than $0.45 \%$, which proves that the radical difference in the computational effort is not reflected in the significant differences in the results between the FE models. Further, since new finite elements have been primarily developed for the purposes of inverse identification, where several input parameters are uncertain, this level of the accuracy should therefore be satisfactorily.

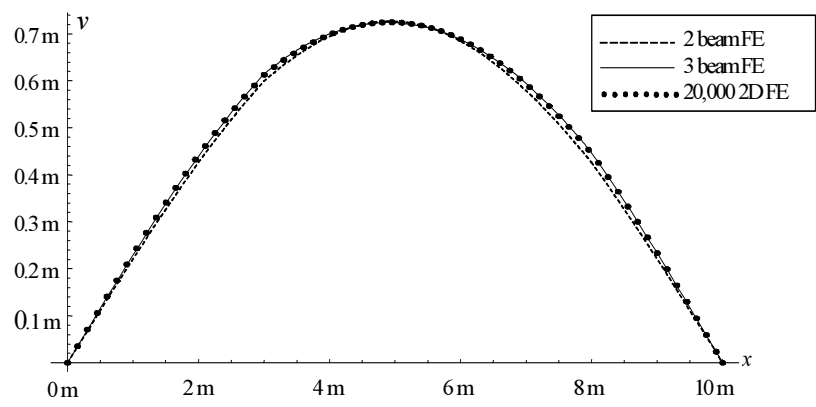

Fig. 5: Transverse displacements $\mathrm{v}$ for second load case

Besides transverse displacements, bending moments and shear forces were also evaluated for both load cases and then compared with the values obtained with elementary static analysis. Since these results exhibited perfect matching they are not presented here.

\section{CONCLUSION}

This paper overcomes the limitation on hinge modeling within the structural element as two new beam finite elements for the analysis of beams with transverse cracks are presented in the paper. Corresponding stiffness matrixes and load vectors, as well the interpolation functions for supplementary computation of transverse displacements within the elements are given in closed symbolic forms. It is obvious that for the uncracked case, i.e. $K_{r}=\infty$, all given results reduces into standard expressions for the noncracked elements.

The main advantage of the presented beam finite elements with transverse cracks over the 2D FE discretisations is clearly evident as their implementation allows the utilization of small and compact, but still reliable computational models. Consequently, essentially less computational effort is required as the transverse displacements, reactions and also inner forces can be computed from a significantly smaller number of linear equations as with proper $2 \mathrm{D}$ finite elements' description of cracks. Since presented stiffness matrixes and load vectors are written entirely in symbolic forms, this allows their straightforward implementation in the existing finite element software 
and consequently, fast real time analysis. Further, since also the corresponding interpolation functions are already presented, this allows additional derivation of mass and geometrical stiffness matrixes for the presented finite elements.

The general drawback of the applied simplified model is the complete absence of information about the stress distribution in the vicinity of the crack, which is essential for crack propagation analysis. However, considering that the main essence of proposed solutions is to yield the best possible global response with the minimum possible data, which is essential from the inverse identification point of view, this is not an essential limitation as non-propagation of cracks during the inverse analysis is assumed.

Although presented finite elements can provide reliable information about transverse displacements, inner forces and reaction, the focus of the future work should be oriented towards the inclusion of shear stresses in the model which should expand the utilization of the computational model also for nonslender elements.

\section{ACKNOWLEDGEMENTS}

This research was supported by the Slovenian Research Agency (ARRS) through the research project »Identification of structures, soils and defects«, J26020-0797-04/2.01.

\section{REFERENCES}

1. Okamura, H., H.W. Liu, C. Chorng-Shin and H. Liebowitz, 1969. A Cracked Column under Compression. Engineering Fracture Mechanics, 1: 547-564.

2. Rizos, P.F., N. Aspraghtas and A.D. Dimarogonas, 1990. Identification of crack location and magnitude in a cantilever beam from the vibration modes, J. of Sound and Vibration, 138: 381-388.

3. Boltežar, M., B. Strancar and A. Kuhelj, 1998. Identification of transverse crack location in flexural vibrations of free-free beams. J. Sound Vibration, 211 (5): 729-734.

4. Skrinar, M. and A. Umek, 1996. Plane finite element of a beam with a transverse crack, Slovenian journal of Civil Engineering/Gradbeni vestnik, $1 / 2: 2-7$.

5. Skrinar, M. 1997. A simple FEM beam element with an arbitrary number of cracks. Internationales Kolloquium über Anwendungen der Informatik und Mechanik in Architektur und Bauwesen IKM, Weimar 1997. Weimar: Universität Weimar.

6. Skrinar, M., 2000. Simple model for the vertical displacements computation of single cracked cantilever under tension loads. Zeitschrift für angewandte Mathematik und Mechanik, 80, suppl. 2: 551-552.
7. Skrinar, M., 2001. On vertical displacements of single cracked beams under tension load application of simple computational model in simply supported beams. Zeitschrift für angewandte Mathematik und Mechanik, 81, suppl. 4: 893-894.

8. Skrinar, M., 2001. End-supported cantilever with a single crack under tension loads. In: Advances in computational engineering \& sciences, ICES'01 (eds S.N. Atluri, T. Nishioka and M. Kikuchi). Tech Science Press, Palmdale, CA

9. Skrinar, M., 2001. On the computation of transverse displacements of slender beam elements with a single sided crack under tensional longitudinal loads. In: 10th International Conference on Computational Methods and Experimental Measurements CMEM X (eds. Y. Villacampa Esteve, G.M. Carlomagno and C.A. Brebbia) pp. 311-320. WIT Press, Southampton; Boston.

10. Skrinar, M., 2003. Dynamical analysis of frame type structures with local stiffness reductions by a new finite element based on the dynamic stiffness and mass matrices. In: 11th International Conference on Computational Methods and Experimental Measurements CMEM XI (eds. C.A. Brebbia, G.M. Carlomagno and P. Anagnostopoulos ) pp. 3-12. WIT Press, Southampton; Boston.

11. Ostachowicz, W.M. and M. Krawczuk, 1990. Vibrational analysis of cracked beam. Computer and Structures, 36(22): 245-250.

12. Liang, R.Y., H. Jialou and F. Choy, 1992. Theoretical Study of Crack-Induced Eigenfrequency Changes on Beam Structures. J. of Engineering Mechanics, 118(2): 384-396.

13. Krawczuk, M. and W.M. Ostachowicz, 1993. Influence of a crack on the dynamic stability of a column. J. of Sound and Vibration 167(3): 541555.

14. Sundermayer, J.N. and R.L. Weaver, 1993. On crack identification and characterization in a beam by nonlinear vibration analysis, Theoretical and applied mechanics, TAM Report No. 743, UILUENG-93-6041.

15. Hasan, W.M., 1995. Crack detection from the variation of the eigenfrequencies of a beam on elastic foundation. Engineering Fracture Mechanics, 52(3): 409-421.

16. Skrinar, M. and T. Pliberšek, 2004. New linear spring stiffness definition for displacement analysis of cracked beam elements. PAMM Proc. Appl. Math. Mech. 4: 654-655. 